\title{
INFRARED SPECTRA OF RETARDED MOLECULAR OSCILLATOR
}

\author{
A. Witkowski* ${ }^{*}$ O. Henri-Rousseau and P. Blaise \\ Centre d'Etudes Fondamentales, Université de Perpignan \\ 52 av. de Villeneuve, 66860 Perpignan cedex, France \\ (Received July 17, 1996; revised version November 6, 1996)
}

Theoretical interpretation is proposed for the inhomogeneous broadening of the infrared line shapes of high harmonics of hydrogen containing modes observed by the optoacoustic and thermal lensing spectroscopy in the liquid phase of benzene and other organic transparent liquids. Attention is focused on the experimental fact that both line width and asymmetry factors of the harmonic series increase linearly with the harmonic number for which fact no satisfactory theoretical understanding was still available. This interpretation is based on recently proposed new approach to separation of electronic and nuclear movements accounting for the finite velocity of the $\pi$ electronic cloud of vicinal molecules and its retardation in following the protonic motion. This retardation leads to the time dependence of molecular oscillator which may be viewed as anharmonic. The experimental results concerning the linear increase in the line width and the asymmetry factor with increasing order of the harmonic are well represented.

PACS numbers: $33.70 . \mathrm{Jg}, 33.20 . \mathrm{Ed}$

\section{Introduction}

Development of new experimental methods in spectroscopy [1] now allows a precise determination of absorption strengths and profiles of high harmonics of vibrational modes, properties unaccessible before by the classical spectroscopic measurements. In particular, the optoacoustic methods [1-3] and thermal lensing spectroscopy $[4,5]$ allowed recently to determine infrared (IR) profiles of high harmonics ( $n=6$ to 8 ) of hydrogen containing modes in transparent liquids (benzene).

Importance of high overtones of hydrogen containing vibrational modes in different fields of photochemistry is well recognized. Highly excited stretching vibrations are the principal accepting modes in the radiationless transitions in polyatomic molecules and recent research activity concerning mode selectivity in unimolecular reactions points out [6] importance of these modes in dissociation of remote bonds and vibrational energy redistribution in weakly bounded complexes.

*Permanent address: Department of Theoretical Chemistry, Jagiellonian University, Ingardena 3, 30-060 Cracow, Poland. 
High overtones of hydrogen containing modes in organic liquids (benzene, naphtalene etc.) involve broadened asymmetric absorption profiles. More precisely, the width and the asymmetry are observed to be linearly increasing with the overtone number. As pointed out in the literature [1], no theoretical understanding of these functional dependences was available and to the best of our knowledge these linear functional dependences remain still unexplained. More interesting is the fact that neither of the two basic classes of the relaxation theories in liquids [7] seems to be applicable to this observation.

On the one hand, the gas-like isolated binary collision models do not seem applicable as in gas phase the absorption profiles are symmetric, narrow and decrease in width with increasing $n$ [2].

On the other hand, the weak coupling theories (weak coupling of the vibrational degrees of freedom to the bath, e.g. translations and rotations) also cannot explain the observed line width dependence on $n$ because dilution with $\mathrm{CCl}_{4}$ (which keeps coupling with the bath unchanged) diminishes the width and makes the asymmetry disappear [1].

Indeed, these spectral properties of organic liquids are intriguing, as compared with the gas phase where profiles are symmetric, narrow and decrease in width with increasing $n$. In view of this sharp contrast, Patel and Tam [1] have concluded that these spectral properties are mainly caused by the proximity of other benzene molecules. In order to explain this sharp contrast the spatial anharmonicity of the $\mathrm{C}-\mathrm{H}$ oscillators can be neglected as there is no reason why this anharmonicity should be different in liquids as compared with the gas phase.

We consider that the reason of the observed linear increase in the line width and asymmetry factor with respect to the harmonic number in pure liquid phase, is another anharmonicity, we shall call it dynamical, which results from some time retardation in the interaction between the proton of the $\mathrm{C}-\mathrm{H}$ bond of a first molecule and the $\pi$ electronic cloud of a neighbor one. Since $\pi$ electrons are weakly bonded, they may be viewed as slow with respect to usual bonding electrons such as the $\sigma$ ones. As a consequence of this slow velocity, some retardation will appear for the $\pi$ electronic cloud of the second molecule in following the motion of the proton involved in the $\mathrm{C}-\mathrm{H}$ bond of the first molecule. This retardation will lead, in the framework of the new approach, to separation of electronic and nuclear movements [8] to write the Hamiltonian of the $\mathrm{C}-\mathrm{H}$ oscillator initially considered as harmonic to be time-dependent and may be viewed after Fourier transform as anharmonic. This dynamical anharmonicity is at the origin of the explanation which we propose for the observed asymmetry and for the linear increase in the asymmetry factor with respect to the harmonic number.

\section{Retarded molecular oscillator}

Recently a new approach to the separation of nuclear and electronic motions in molecular quantum mechanics has been proposed [8] accounting for the finite velocity of an electron and its retardation in following the nuclear motions.

In the standard Born-Oppenheimer approximation the potential energy for the nuclear movement (in appropriate dimensionless units)

$$
E(q)=(1 / 2) q^{2}
$$


results from immediate electronic adjustment to any nuclear position (electron "sees" only the nuclear position but no nuclear velocity). The real delay in adjustment compared with the $q$ coordinate in (1) is simply given by $\widetilde{q}$ (in dimensionless units, and with time units $\left.t=\omega t^{\prime}\right)$

$$
\tilde{q}=q+(\dot{q} / v) t=q+\kappa p t,
$$

where $v$ is the mean electronic velocity

$$
v=\left\langle\Psi\left|p_{\mathrm{e}} / m\right| \Psi\right\rangle,
$$

$\omega$ is the harmonic frequency, $\dot{q}-$ the nuclear velocity and

$$
\kappa=\omega / v
$$

can be evaluated $[8]$ from the Heisenberg relation

$$
\hbar \kappa=\left\langle p_{\mathrm{e}}\right\rangle(m / M)^{1 / 2}
$$

where $\left\langle p_{\mathrm{e}}\right\rangle$ is the mean electronic linear momentum.

An interaction representation for separation of nuclear and electronic motions accounting for the time dependent nuclear coordinates (2) leads [8] to the following Hamiltonian of a linear molecular oscillator:

$$
\eta(t)=(1 / 2)\left(p^{2}+q^{2}\right)+(1 / 2)(p q+q p) \kappa t+(1 / 2)(\kappa t)^{2} p^{2},
$$

where $t=\omega t^{\prime}$ and $[p, q]=-\mathrm{i}$.

For mean electronic velocity $v \rightarrow \infty, \kappa \rightarrow 0$ and (6) reduces to its standard Born-Oppenheimer form.

To determine the IR spectra of an oscillator described by Hamiltonian (6), it is convenient to write (6) in a different form resulting from transition to the creation and destruction operators

$$
\begin{aligned}
& p=(\mathrm{i} / \sqrt{2})\left(a^{\dagger}-a\right), \quad q=(1 / \sqrt{2})\left(a^{\dagger}+a\right), \\
& {\left[a, a^{\dagger}\right]=1 .}
\end{aligned}
$$

Then Eq. (6) can be written in an equivalent form

$$
\eta(t)=\left[1+(1 / 2)(\kappa t)^{2}\right] \tau_{3}+(\kappa t) \tau_{2}-(1 / 2)(\kappa t)^{2} \tau_{1},
$$

where

$$
\begin{aligned}
\tau_{1} & =(1 / 2)\left[a^{\dagger} a^{\dagger}+a a\right], \\
\tau_{2} & =(\mathrm{i} / 2)\left[a^{\dagger} a-a a\right], \\
\tau_{3} & =(1 / 2)\left[a^{\dagger} a+a a^{\dagger}\right]=a^{\dagger} a+1 / 2 .
\end{aligned}
$$

Here $\tau_{i}$ are operators obeying the following commutation rules:

$$
\begin{aligned}
& {\left[\tau_{1}, \tau_{2}\right]=2 \mathrm{i} \tau_{3},} \\
& {\left[\tau_{2}, \tau_{3}\right]=-2 \mathrm{i} \tau_{1},} \\
& {\left[\tau_{3}, \tau_{1}\right]=-2 \mathrm{i} \tau_{2} .}
\end{aligned}
$$

From (9), (13) and (15) follow the equations of motion for the $\tau_{i}$ operators

$$
\dot{\tau}_{1}=2\left[1+(1 / 2)(\kappa t)^{2}\right] \tau_{2}+2 \kappa t \tau_{3}
$$




$$
\begin{aligned}
& \dot{\tau}_{2}=-2\left[1+(1 / 2)(\kappa t)^{2}\right] \tau_{1}+2(\kappa t)^{2} \tau_{3} \\
& \dot{\tau}_{3}=2 \kappa t \tau_{1}+(\kappa t)^{2} \tau_{2} .
\end{aligned}
$$

Equations (16)-(18) now allow us to diagonalize (9) by the method of successive approximations. For this aim we put; for $t=0, \tau_{1}(0)=\tau_{2}(0)=0$ and $\tau_{3}(0) \neq 0$. Then from Eqs. (16)-(18), we obtain respectively

$$
\begin{aligned}
& \dot{\tau}_{1}=2 \kappa t \tau_{3}(0), \quad \tau_{1}=\kappa t^{2} \tau_{3}(0), \\
& \dot{\tau}_{2}=(\kappa t)^{2} \tau_{3}(0), \quad \tau_{1}=(1 / 3) \kappa^{2} t^{3} \tau_{3}(0),
\end{aligned}
$$

and in the first step of approximation we have from (9)

$$
\eta^{(1)}(t)=\left[1+(1 / 2) \kappa^{2} t^{2}-(1 / 6) \kappa^{3} t^{4}\right] \tau_{3}(0)=\left[1+f_{1}(k, t)\right] \tau_{3}(0) .
$$

Then, in the next step, from Eqs. (12), (16), (19) and (20), we have for Eq. (9)

$$
\eta^{(2)}(t)=\left[1+(1 / 2) \kappa^{2} t^{2}-L\left(t^{4}, t^{6}, t^{8}\right)\right] \tau_{3}(0)
$$

where in $L$ are the terms of fourth and higher orders in $t$, and in this way we may diagonalize (9) to any desired order of approximation.

As the potential energy in Eq. (1) is harmonic and transformation to the delayed coordinate (2) linear in time, therefore it is reasonable to retain in Hamiltonian (22) terms quadratic in time and neglect those of higher orders.

Hamiltonian (6) can be also diagonalized by the standard Bogolyubov method which we did before in another context [9]. We use here the method of successive approximations in terms of the Lie algebra of the $\mathrm{SO}(2,1)$ group which has here an advantage over the Bogolyubov method.

For $|\kappa|<1$, it leads to an analytical form of the spectral density and the same procedure in terms of anothenrealization of the Lie algebra of $\mathrm{SO}(2,1)$ group allows us to diagonalize the electronic interactions [8]. It makes also clear that for $|\kappa|>1$, instead of $\tau_{3}$ the $\tau_{1}, \tau_{2}$ operators should be diagonalized which leads to the new coherent states [10].

\section{The infrared absorption spectrum}

The infrared absorption line-shape function $I(\Omega)$ is the Fourier transform of the autocorrelation function of the dipole moment operator $\mu(t)$, which, in appropriate units, equals the nuclear coordinate $\tilde{q}(t)$

$$
I(\Omega)=\int_{-\infty}^{+\infty} \mathrm{d} t \exp (\mathrm{i} \Omega t)\langle\mu(0) \mu(t)\rangle .
$$

Define now the eigenfunctions $|n\rangle$ by

$$
(1 / 2)\left(p^{2}+q^{2}\right)|n\rangle=\tau_{3}|n\rangle=(n+1 / 2)|n\rangle
$$

in $\hbar \omega$ units. Then, let us look at the time dependence of dipole moment transition. We may write

$$
\operatorname{id}[\langle 0|q| n\rangle] / \mathrm{d} t=\langle 0|q| n\rangle\left\langle n\left|\eta^{(i)}(t)\right| n\right\rangle-\left\langle 0\left|\eta^{(i)}(t)\right| 0\right\rangle\langle 0|q| n\rangle .
$$

Therefore from (22) (where we put $L=0$ ) we have

$$
\langle 0|q(t)| n\rangle=\langle 0|q(0)| n\rangle \exp \left\{-\mathrm{in} \omega \int_{0}^{t}\left[1+(1 / 2) \kappa^{2} t^{2}\right] \mathrm{d} t\right\}
$$


and, in a similar way

$$
\langle 0|p(t)| n\rangle=\langle 0|p(0)| n\rangle \exp \left\{-\mathrm{in} \omega \int_{0}^{t}\left[1+(1 / 2) \kappa^{2} t^{2}\right] \mathrm{d} t\right\} .
$$

Next let us in (23) denote

$$
F_{n}(\kappa, \Omega)=\int_{-\infty}^{+\infty} \mathrm{d} t \exp (\mathrm{i} \Omega t)\langle 0|\mu(t)| n\rangle .
$$

Then we have

$$
I(\kappa, \Omega)=\left|F_{n}(\kappa, \Omega)\right|^{2}
$$

with, in view of Eq. (2),

$$
F_{n}(\kappa, \Omega)=\int_{-\infty}^{+\infty} \mathrm{d} t \exp (\mathrm{i} \Omega t)[\langle 0|q(t)| n\rangle+\kappa t\langle 0|p(t)| n\rangle] .
$$

Again, by aid of Eqs. (26a), (26b), Eq. (28) takes the form

$$
\begin{gathered}
F_{n}(\kappa, \Omega)=\langle 0|q(0)| n\rangle \int_{-\infty}^{+\infty} \mathrm{d} t \exp (\mathrm{i} \Omega t) \exp \left\{-\mathrm{i} n\left[t+(1 / 6) \kappa^{2} t^{3}\right]\right\} \\
+\kappa\langle 0|p(0)| n\rangle \int_{-\infty}^{+\infty} \mathrm{d} t t \exp (\mathrm{i} \Omega t) \exp \left\{-\mathrm{i} n\left[t+(1 / 6) \kappa^{2} t^{3}\right]\right\}
\end{gathered}
$$

Now, we may observe that

$$
\langle 0|p(0)| n\rangle
$$

appearing in Eq. (29) may be expressed in term of $\langle 0|q(0)| n\rangle$, as follows. First, for time $t=0 \mathrm{Eq}$. (6) reduces to

$$
\eta(0)=(1 / 2)\left[p^{2}(0)+q^{2}(0)\right] .
$$

Again, the commutator of $q$ and $\eta(0)$ is

$$
[q(0), \eta(0)]=\mathrm{i} p(0) \text {. }
$$

Next, we may consider the matrix transition elements of Eq. (32) between the ground state $\langle 0|$ and the $n$-th excited state $|n\rangle$ of $\eta(0)$, i.e.

$$
\langle 0|[q(0), \eta(0)]| n\rangle=\mathrm{i}\langle 0|p(0)| n\rangle \text {. }
$$

This leads to

$$
\mathrm{i}\langle 0|p(0)| n\rangle=\sum[\langle 0|q| m\rangle\langle m|\eta(0)| n\rangle-\langle 0|\eta(0)| m\rangle\langle m|q| n\rangle]
$$

and thus,

$$
\mathrm{i}\langle 0|p(0)| n\rangle=n\langle 0|q(0)| n\rangle .
$$

As a consequence of Eq. (33), Eq. (29) becomes

$$
\begin{gathered}
F_{n}(\kappa, \Omega)=\langle 0|q(0)| n\rangle\left[\int_{-\infty}^{+\infty} \mathrm{d} t \exp (\mathrm{i} \Omega t) \exp \left\{-\mathrm{i} n\left[t+(1 / 6) \kappa^{2} t^{3}\right]\right\}\right. \\
\left.-\mathrm{i} n \kappa \int_{-\infty}^{+\infty} \mathrm{d} t t \exp (\mathrm{i} \Omega t) \exp \left\{-\mathrm{i} n\left[t+(1 / 6) \kappa^{2} t^{3}\right]\right\}\right]
\end{gathered}
$$


or

$$
\begin{aligned}
& F_{n}(\kappa, \Omega)=2\langle 0|q(0)| n\rangle\left[\int_{0}^{+\infty} \mathrm{d} t \cos \left\{n\left[(1 / 6) \kappa^{2} t^{3}+\Omega^{\prime} t\right]\right\}\right. \\
& \left.\quad+n \kappa \int_{0}^{+\infty} \mathrm{d} t t \sin \left\{n\left[(1 / 6) \kappa^{2} t^{3}+\Omega^{\prime} t\right]\right\}\right]
\end{aligned}
$$

where

$$
\Omega^{\prime}=n-\Omega / \omega .
$$

Now, we may observe that the first integral involved in the Fourier transform (35) is of the form [11]:

$$
\int_{0}^{+\infty} \cos \left(a t^{3} \pm X t\right) \mathrm{d} t=\pi(3 a)^{-1 / 3} \mathrm{Ai}\left[ \pm(3 a)^{-1 / 3} X\right]
$$

where $\mathrm{Ai}[x]$ is the Airy function. Besides, we may observe that the second integral involved in Eq. (35) may be viewed as the derivative of the first one

$$
\int_{0}^{+\infty} t \sin \left(a t^{3} \pm X t\right) \mathrm{d} t=-\mathrm{d}\left[\int_{0}^{+\infty} \cos \left(a t^{3} \pm X t\right) \mathrm{d} t\right] / \mathrm{d} X
$$

in view of these remarks we may write

$$
\left.\int_{0}^{+\infty} \mathrm{d} t \cos \left\{n\left[(1 / 6) \kappa^{2} t^{3}+\Omega^{\prime} t\right]\right\}=\pi\left[(1 / 2) n \kappa^{2}\right]^{-1 / 3} \mathrm{Ai}\left[(1 / 2) n \kappa^{2}\right]^{-1 / 3} \Omega^{\prime}\right]
$$

and

$$
\int_{0}^{+\infty} \mathrm{d} t t \sin \left\{n\left[(1 / 6) \kappa^{2} t^{3}+\Omega^{\prime} t\right]\right\}=-\pi\left[(1 / 2) n \kappa^{2}\right]^{-2 / 3} \mathrm{Ai}^{\prime}\left\{\left[(1 / 2) n \kappa^{2}\right]^{-1 / 3} \Omega^{\prime}\right\}
$$

where $\mathrm{Ai}^{\prime}$ denotes the derivative of the Airy function.

As a consequence, with the use of Eqs. (39) and (40), Eq. (35) takes the form

$$
\begin{aligned}
& F_{n}\left(\kappa, \Omega^{\prime}\right)=\langle 0|q(0)| n\rangle 2 \pi\left\{\left[(1 / 2) n \kappa^{2}\right]^{-1 / 3} \mathrm{Ai}\left\{\left[(1 / 2) n \kappa^{2}\right]^{-1 / 3} \Omega^{\prime}\right\}\right. \\
& \left.\quad+n \kappa\left[(1 / 2) n \kappa^{2}\right]^{-2 / 3} \mathrm{Ai}^{\prime}\left\{\left[(1 / 2) n \kappa^{2}\right]^{-1 / 3} \Omega^{\prime}\right\}\right\}
\end{aligned}
$$

Therefore, the spectral density is

$$
\begin{aligned}
& I_{n}\left(\kappa, \Omega^{\prime}\right)=|\langle 0|q(0)| n\rangle|^{2} 4 \pi^{2}\left\{\left[(1 / 2) n \kappa^{2}\right]^{-2 / 3} \mathrm{Ai}^{2}\left\{\left[(1 / 2) n \kappa^{2}\right]^{-1 / 3} \Omega^{\prime}\right\}\right. \\
& \left.\quad+(\kappa n)^{2}\left[(1 / 2) n \kappa^{2}\right]^{-4 / 3} \mathrm{Ai}^{2}\left\{\left[(1 / 2) n \kappa^{2}\right]^{-1 / 3} \Omega^{\prime}\right\}\right\}
\end{aligned}
$$

\section{Practical considerations}

It may be observed that the spectral density as given by Eq. (42) is involving a divergence for high frequencies because of the presence of the first derivative $\mathrm{Ai}^{\prime}$ of the Airy function. Besides, our calculations with the use of Eq. (42) account also only for the inhomogeneous part of the spectral broadening (leaving therefore as constant background of the usual small symmetric broadening observed [1] at the infinite dilution of benzene by an inert solvent $\left(\mathrm{CCl}_{4}\right)$ ). Therefore, in view of this 
last remark, we may introduce a finite natural lifetime through a phenomenological damping parameter $\gamma$, in order to remove the divergence related to the derivative of the Airy function.

More precisely, in place of the Fourier transform $F_{n}(\kappa, \Omega)$ given by Eq. (30) we shall consider another one $F_{n}(\kappa, \gamma, \Omega)$ involving damping and given by

$$
\begin{gathered}
F_{n}(\kappa, \gamma, \Omega)=\langle 0|q(0)| n\rangle\left\{\int_{-\infty}^{+\infty} \mathrm{d} t \exp (\mathrm{i} \Omega t) \exp \left\{-\mathrm{i} n\left[t+(1 / 6) \kappa^{2} t^{3}\right]\right\} \exp (-\gamma t)\right. \\
\left.-\mathrm{i} n \kappa \int_{-\infty}^{+\infty} \mathrm{d} t \exp (\mathrm{i} \Omega t)\left\{t \exp \left\{-\mathrm{i} n\left[t+(1 / 6) \kappa^{2} t^{3}\right]\right\} \exp (-\gamma t)\right\}\right\} .
\end{gathered}
$$

This Fourier transform will be performed numerically, leading then to the spectral density through

$$
I_{n}(\kappa, \gamma, \Omega)=\left|F_{n}(\kappa, \gamma, \Omega)\right|^{2} .
$$

\section{Comparison of experimental and theoretical spectra}

Figure 1 presents the measured absorption profiles at $n=6$ to $n=8$ overtones of $\mathrm{CH}$ stretch in liquid benzene obtained by the laser optoacoustic spectroscopy [2]. Then, Fig. 2 gives that obtained for deuterated benzene- $d_{5}$, for $n=6$
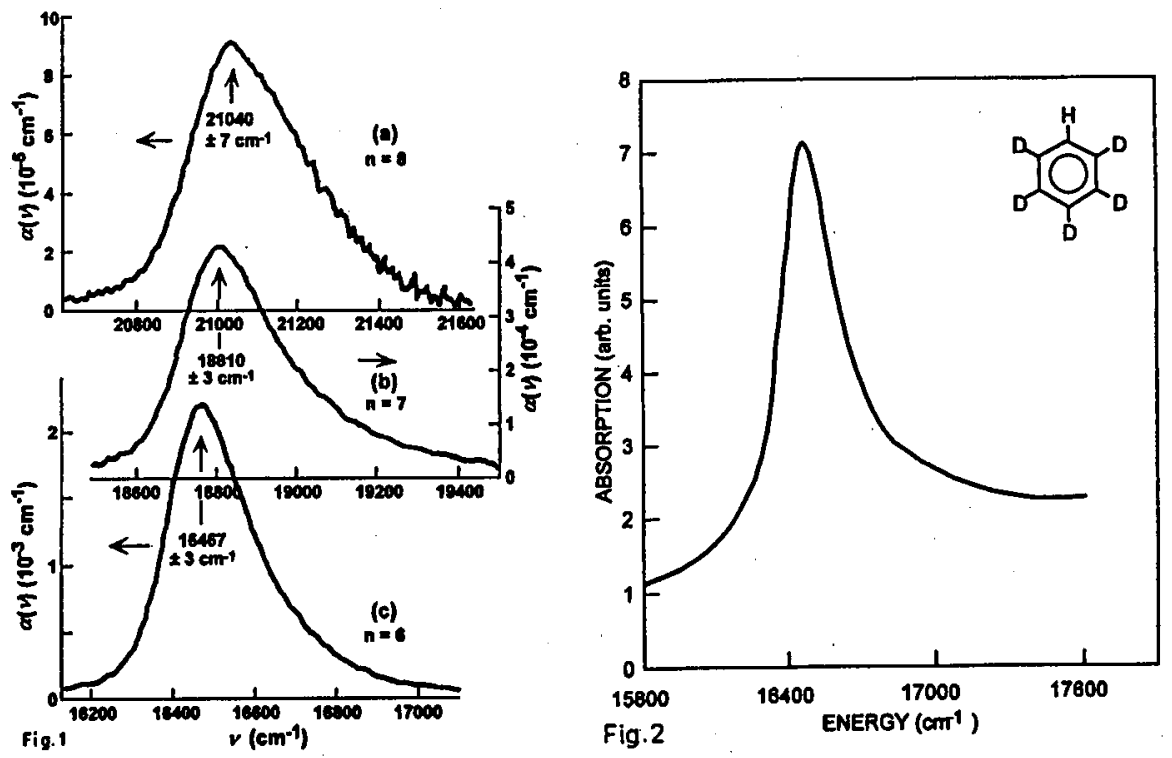

Fig. 1. Measured absorption profiles of the $n=8$ to $n=6$ harmonics of the $\mathrm{C}-\mathrm{H}$ stretch in liquid benzene by laser optoacoustic spectroscopy (from Ref. [2], Fig. 1 with the permission of the authors).

Fig. 2. Thermal lensing absorption spectrum of benzene- $d_{5}$ (from Ref. [5], Fig. 2 with the permission of the authors). 

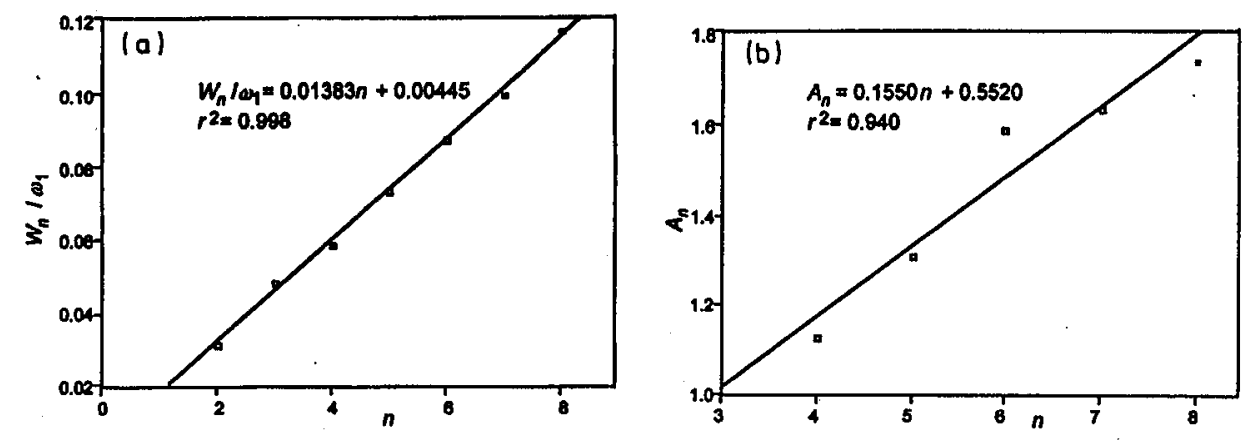

Fig. 3. (a) Ratio $W_{n} / \omega$ of experimental line width (full line width at half maximum on the fundamental frequency $\omega$ as a function of $n$ for the $n$-th harmonic of the $\mathrm{C}-\mathrm{H}$ stretch in liquid benzene (from the data in Ref. [2], Table I). (b) Asymmetry factor $A_{n}$ (ratio of the half-width on the high frequency side of the peak to the half-width on the low frequency side of the peak) versus the $n$-th harmonic of the $\mathrm{C}-\mathrm{H}$ stretch in liquid benzene (from the data in Ref. [2], p. 1472).
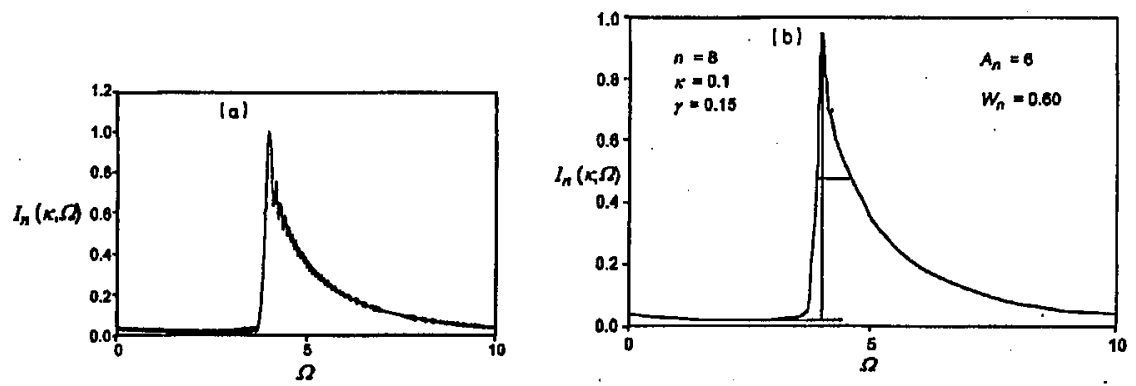

Fig. 4. (a) Calculated spectral density $I_{n}(\kappa, \Omega)$ versus frequency $\Omega$ (in $\omega$ units); (b) Calculated spectral density $I_{n}(\kappa, \Omega)$ versus frequency $\Omega$ (in $\omega$ units) after smoothing the curve.

by the thermal lensing spectroscopy [5]. Very characteristic asymmetry of the absorption profiles for $\mathrm{CH}$ stretch and the local mode character of these vibrations are clearly visible.

Figure 3a presents the observed line width $W_{n}$ (full width at half maximum divided by the fundamental frequency $\omega_{1}=3054 \mathrm{~cm}^{-1}$ ) and Fig. $3 \mathrm{~b}$ gives the asymmetry factor $A_{n}$ (ratio of the half-width on the high frequency side of the peak to the half-width on the low frequency side) for the $n$-th harmonic of $\mathrm{CH}$ stretch in liquid benzene as a function of $n$. The displayed dependence is clearly a linear one.

The theoretical IR absorption profiles for $n=6,7,8$ were calculated for $\kappa=0.1$ (in $\hbar \omega$ units) and $\gamma / \omega=0.15$, from (43) and (44). In Fig. 4a is given an example of band profile for $n=8$ and in Fig. $4 \mathrm{~b}$ is shown the same profile after smoothing the numerical curve by a standard procedure, in order to make easier the measure of the line width. 

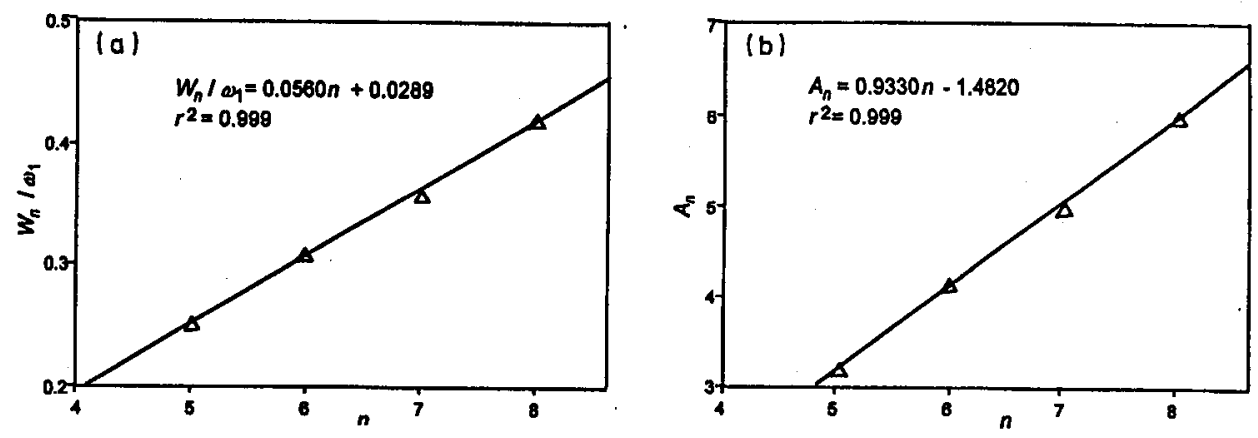

Fig. 5. (a) Ratio $W_{n} / \omega$ of theoretical line width (full line width at half maximum on the fundamental frequency $\omega$ ) as a function of the $n$-th harmonic; (b) Theoretical asymmetry factor $A_{n}$ (ratio of the half-width on the high frequency side of the peak to the half-width on the low frequency side of the peak) versus the $n$-th harmonic.

Figure 5a shows the linear regression versus $n$ of the theoretical line widths $W_{n}$ (full width at half maximum divided by the fundamental frequency $\omega=1$ ) and Fig. 5b the corresponding theoretical asymmetry ratio $A_{n}$, for $n=5$ to 8 .

As it appears, the theory is well reproducing the experimental linear dependences on $n$ of the line width and of the asymmetry.

\section{Conclusion}

The main results of the spectral observations, i.e. the linear increase with the overtone number of the asymmetry and width of the absorption profiles were therefore reproduced by our model of a retarded molecular oscillator. This reproduction was obtained as function of the retardation parameter $\kappa$, whose numerical value was not calculated here theoretically. This, by (5), would require a very high quality of the electronic wave function to obtain the average electronic velocity value. There is a much more convincing argument for the validity of our model: isotopic substitution of hydrogen by deuterium which, by (5), changes the parameter $\kappa$ and modifies in an appropriate way the theoretical linear dependence. Unfortunately, we were not able to find the corresponding deuterated spectra in the literature.

New experiments in the same conditions as in Refs. $[1,2]$, but with deuterium substituted species would provide results very helpful for understanding the nature of the observed spectral behavior.

\section{References}

[1] C.K.N. Patel, A.C. Tam, Rev. Mod. Phys. 53, 517 (1981).

[2] C.K.N. Patel, A.C. Tam, R.J. Kerl, J. Chem. Phys. 71, 1470 (1979).

[3] C.K.N. Patel, E.T. Nelson, R.J. Kerl, Nature 286, 368 (1980).

[4] R.L. Swofford, M.E. Long, A.C. Albrecht, J. Chem. Phys. 65, 179 (1976).

[5] R.L. Swofford, M.S. Burberry, J.A. Morell, A.C. Albrecht, J. Chem. Phys. 66, 52 (1977). 
[6] T. Uzer, J.T. Agnes, Chem. Phys. 139, 163 (1989).

[7] D.W. Oxtoby, Adv. Chem. Phys. 47, 487 (1981).

[8] A. Witkowski, Phys. Rev. A 41, 3511 (1990).

[9] A. Witkowski, J. Chem. Phys. 79, 852 (1983).

[10] A.O. Barut, L. Girardello, Commun. Math. Phys. 21, 41 (1971).

[11] Handbook of Mathematical Functions, Eds. M. Abramowitz, I.A. Stegun, Nat. Bur. Stand., US. GPO, Washington 1964. 